\title{
TFAP2C promotes stemness and chemotherapeutic resistance in colorectal cancer via inactivating hippo signaling pathway
}

Xu Wang ${ }^{1+}$, Di Sun ${ }^{1+}$, Jiandong Tai ${ }^{1}$, Si Chen ${ }^{1}$, Miao Yu², Dong Ren $^{3}$ and Lei Wang ${ }^{1 *}$

\begin{abstract}
Background: Aberrant expression of transcription Factor AP-2 Gamma (TFAP2C) has been reported to be implicated in malignant process of many cancers. The purpose of this study is to investigate the clinical significance and biological roles of TFAP2C in colorectal cancer (CRC).

Methods: TFAP2C expression was evaluated by real-time PCR, Western blot and immunohistochemistry (IHC) respectively in clinical CRC tissues. Statistical analysis was performed to explore the correlation between TFAP2C expression and clinicopathological features, and overall and progression-free survival in CRC patients. In vitro and in vivo assays were performed to assess the biological roles of TFAP2C in CRC cells. Western blot, luciferase and Chromatin immunoprecipitation (ChIP) assays were used to identify the underlying pathway mediating the biological roles of TFAP2C in CRC.

Results: TFAP2C is robustly upregulated in CRC tissues and cells, and high expression of TFAP2C correlates with advanced clinicopathological features, poor prognosis and disease progression in CRC patients. Furthermore, upregulating TFAP2C enhances spheroids formation ability, the fraction of SP cells, expression of stem cell factors and the mitochondrial potential, and reduces the apoptosis induced by 5 -fluorouracil in colorectal cancer cells in vitro, and promotes stemness and chemoresistance of CRC cells in vivo; while silencing TFAP2C yields an opposite effect. Importantly, downregulation of TFAP2C dramatically restores chemotherapeutic sensitivity of CRC cells to 5-FU in vivo. Our results further demonstrate that TFAP2C promotes stemness and chemoresistance of CRC cells to 5-FU by inhibiting Hippo signaling via transcriptionally upregulating ROCK1 and ROCK2 in CRC cells.

Conclusion: Our findings indicate that TFAP2C may serve as a novel prognostic factor in CRC patients, and a therapeutic target for the treatment of CRC, suggesting that silencing TFAP2C in combination with 5-FU may be an effective therapeutic strategy to improve survival in CRC patients.
\end{abstract}

Keywords: TFAP2C, Chemotherapeutic resistance, Cancer stem cells, Hippo signaling and colorectal cancer

\footnotetext{
* Correspondence: wanglei20160714@163.com

${ }^{\dagger}$ Equal contributors

'Department of Colorectal and Anal Surgery, The First Hospital of Jilin

University, 71 Xinmin Street, Changchun, Jilin 130000, People's Republic of

China

Full list of author information is available at the end of the article
} 


\section{Background}

Colorectal cancer $(\mathrm{CRC})$ is the most common malignant cancer in digestive tract worldwide $[1,2]$. Despite great progress in treatment of CRC, including 5-fluorouracil (5FU)-based chemotherapy, the combination strategies of 5-FU and oxaliplatin or irinotecan, the five-year survival rate is still dismal $[3,4]$. The existence of cancer stem cells (CSCs) that are the minority population of cells characterized by the capabilities of self-renewal, unlimited proliferation and differentiation into the multiple lineages of cancer cells has been regarded to be responsible for the failure of chemotherapy in CRC patients $[5,6]$, which contributes to the poor prognosis of CRC patients [7]. Indeed, multiple studies have shown that CSCs play crucial roles in the induction and maintenance of chemotherapeutic resistance in several human cancers $[8,9]$. Thus, improved understanding of the mechanisms that maintain CSCs properties will improve the efficacy of chemotherapy in patients with CRC via eradicating the CSC population.

Activator protein-2 (AP-2) factors are a conserved family of DNA-binding transcription factors in different species and five members have been identified in mammals, including AP- $2 \alpha-\varepsilon[10]$. AP-2 factors play important roles in embryogenesis, and interestingly AP-2 factors expression is hardly detectable in most adult tissues $[11,12]$. However, overexpression of AP-2 factors has been observed in multiple human cancers. For example, TFAP2A expression was elevated in nasopharyngeal carcinoma, which promoted nasopharyngeal carcinoma proliferation and growth via inducing cyclooxygenase-2 expression [13]; in addition, TFAP2B was reported to be overexpressed in non-small cell lung cancer (NSCLC) tissues and cell lines. TFAP2B knockdown by siRNA significantly attenuated cell growth and induced apoptosis in NSCLC cells in vitro and in a lung cancer subcutaneous xenograft model; conversely, upregulation of TFAP2B yielded an opposite effect [14]. Furthermore, accumulating literatures have shown that AP-2 proteins play crucial roles in the development of therapeutic resistance in the treatment of cancers. TFAP2A was elevated in breast cancer tissue and cell lines and more highly expressed in tamoxifen resistant tumor tissues and cell lines [15]. High expression of TFAP2C in breast cancer contributed to hormone resistance, which positively correlated with poor survival in breast cancer patients [16]. Moreover, low expression of TFAP2E due to hypermethylation was significantly associated with nonresponse to chemotherapy in colorectal cancer [17]. Therefore, these studies have indicated that different members of AP-2 proteins have stimulatory or inhibitory affect on chemotherapeutic response in cancer treatment, which may be environment and tumor type dependent.

The Hippo signaling pathway has been reported to be dysregulated in several biological processes and tumorigenesis of multiple human cancers [18-20]. In the mammal, the Hippo pathway constitutes of four core kinase cassette components, including kinases MST1/2 and LATS1/2, as well as the adaptor proteins SAV1 and MOB1 [20]. The Hippo signaling is active via tightly balancing the activity of YAP and TAZ, to low levels through phosphorylation- ubiquitination mechanisms [21, 22]. While Hippo signaling is absent, unphosphorylated YAP1/ TAZ enters the nucleus and induce the transcriptional activity of TEA domain (TEAD) family members (TEAD1TEAD4) as the transcriptional co-activators, which further transcriptionally upregulate multiple downstream effectors to exert a pleiotropic role in tumor progression and metastasis [23-25]. Moreover, accumulating studies has shown that the inactivation of Hippo signaling rendered resistance of cancer cells to chemotherapeutic drugs in various types of cancers. Chen et al. reported that simultaneous downregulation of MST1, LATS2, MOB1 and SAV1 by upregulation of miR-183c contributed to chemoresistance in pancreatic cancer [26]; in addition, the study by Touil and colleagues showed that hyperactivation of YAP promoted resistace of colon cancer cells to 5-FU [27]. Accordingly, the inactivation of Hippo pathway is considered as a crucial mediator in development of cancer chemoresistance, and better understanding of the mechanisms underlying the inactivation of Hippo pathway may provide new insights for the development of more effective cancer therapy.

In this study, we find that TFAP2C is significantly upregulated in CRC tissues and cells and high expression of TFAP2C correlates with advanced clinicopathological features, poor prognosis and disease progression in CRC patients. Furthermore, upregulation of TFAP2C enhances, while silencing TFAP2C inhibits CSCs characteristics and chemotherapeutic resistance in CRC cells in vitro and in vivo. Our results further reveal that TFAP2C promotes CSCs characteristics and chemoresistance via transcriptionally activating negative regulators of Hippo signaling, ROCK1 and ROCK2, resulting in inactivation of Hippo signaling in CRC cells. Therefore, our findings identify TFAP2C as a prognostic factor for CRC patients, as well as a therapeutic target to attenuate chemoresistance of CRC.

\section{Methods}

\section{Cell lines and cell culture}

The normal colon epithelial cell CMEC was purchased from PriCells, and all colorectal cancer cell lines, including RKO, CW-2, SW948, HCT116, SW480 COLO 210DM and COLO 205, were obtained from Shanghai Chinese Academy of Sciences cell bank (China). All were cultured in RPMI-1640 medium (Life Technologies, Carlsbad, CA, US) supplemented with penicillin G $(100 \mathrm{U} / \mathrm{ml})$, streptomycin $(100 \mathrm{mg} / \mathrm{ml})$ and 10\%fetal bovine serum (FBS, Life 
Technologies) and cultured at $37{ }^{\circ} \mathrm{C}$ in a humidified atmosphere with $5 \% \mathrm{CO}_{2}$.

\section{Patients and tumor tissues}

A total of eight paired fresh colorectal cancer tissues with matched adjacent normal tissues and individual 378 paraffin-embedded, archived CRC tissues were obtained during surgery at the The First Hospital of Jilin University (Changchun, China) between January 2008 and December 2011 (Additional file 1: Table S1 and Additional file 2: Table S2). Patients were diagnosed based on clinical and pathological evidence, and the specimens were immediately snap-frozen and stored in liquid nitrogen tanks. For the use of these clinical materials for research purposes, prior patients' consents and approval from the Institutional Research Ethics Committee were obtained.

\section{RNA extraction, reverse transcription, and real-time PCR} Total RNA from tissues or cells was extracted using TRIzol (Life Technologies) according to the manufacturer's instructions. Messenger RNA (mRNA) were polyadenylated using a poly-A polymerase-based First-Strand Synthesis kit (TaKaRa, DaLian, China) and reverse transcription (RT) of total mRNA was performed using a PrimeScript RT Reagent kit (TaKaRa) according to the manufacturer's protocol. Complementary DNA (cDNA) was amplified and quantified on ABI 7500HT system (Applied Biosystems, Foster City, CA, USA) using SYBR Green I (Applied Biosystems). Additional file 3: Table S3 lists the primers used in the reactions. Real-time PCR was performed according to a standard method, as described previously [28]. Primers for TFAP2C were synthesized and purified by RiboBio (Guangzhou, China). Glyceraldehyde-3-phosphate dehydrogenase (GAPDH) was used as endogenous controls for miRNA or mRNA respective. Relative fold expressions were calculated with the comparative threshold cycle $\left(2^{-\Delta \Delta C t}\right)$ method according to the previous study [29].

\section{Plasmid, small interfering RNA and transfection}

Human TFAP2C cDNA was purchased form (Vigene Biosciences, Shandong, China) and cloned into the pSin-EF2 plasmid (addgene \#16578, Cambridge, MA, USA). Knockdown of endogenous TFAP2C was performed by cloning two short hairpin RNA (shRNA) oligonucleotides into the pSUPER-puro-retro vector (OligoEngine, Seattle, WA, USA). The full sequence and two separate shRNA fragments of TFAP2C are listed in Additional file 4: Table S4. The luciferase reporter system of pGL6-TA promoter-luc was used to examine the transcriptional activity of ROCK1 and ROCK2, and the sequences of ROCK1 and ROCK2 promoter was presented in Additional file 4: Table S4. Small interfering RNA (siRNA) for YAP and TAZ knockdown was obtained from Ribobio (Guangzhou, China). Transfection of siRNAs and plasmids was performed using Lipofectamine 3000 (Life Technologies) according to the manufacturer's instructions.

\section{Western blotting analysis}

Nuclear/cytoplasmic fractionation was separated by using Cell Fractionation Kit (Cell Signaling Technology, USA) according to the manufacturer's instructions, and the whole cell lysates were extracted using RIPA Buffer (Cell Signaling Technology). Western blot was performed according to a standard method, as described previously [30]. Antibodies against TFAP2C, BCL2 and BCL2L1, p-MST1/2, p-LATS1, LATS1, p-YAP, YAP, ROCK1 and ROCK2 were purchased from Cell Signaling Technology (Cambridge, USA), and MST1 and TAZ from Abcam. The membranes were stripped and reprobed with an anti- $\alpha$-tubulin antibody (Cell Signaling Technology) as the loading control.

\section{Chromatin immunoprecipitation (ChIP)}

Cells were cultured as described above. Cross-linking was performed with formaldehyde (Merck) at a final concentration of $1 \%$ and terminated after 5 min by the addition of glycine at a final concentration of $0.125 \mathrm{M}$. Cells were harvested with SDS buffer, pelleted by centrifugation, and resuspended in IP buffer. Chromatin was sheered by sonication (HTU SONI 130; Heinemann) to generate DNA fragments with an average size of $500 \mathrm{bp}$. Preclearing and incubation with anti-Flag (F1804, Sigma) antibodies or IgG control (M-7023, Sigma) for $16 \mathrm{~h}$ was performed as previously described (Menssen et al. 2007). Washing steps and the reversal of cross-linking were performed as described previously (Frank et al. 2001). Immunoprecipitated DNA was analyzed by qPCR and the primers of ROCK1 and ROCK2 promoter was presented in Additional file 3: Table S3. Enrichment was expressed as the percentage of the input for each condition.

\section{Anchorage-independent growth assay}

A total of 500 cells were trypsinized and suspended in $2 \mathrm{ml}$ of complete medium containing $0.3 \%$ agar (Sigma). This experiment was performed as previously described [31] and carried out three times independently for each cell line.

\section{Cell counting Kit-8 analysis}

For cell counting kit- 8 analysis, cells $\left(2 \times 10^{3}\right)$ were seeded into 96 well plates and the specific staining process and methods were performed according to the previous study [32].

\section{Colony formation assay}

The cells were trypsinized as single cell and suspended in the media with $10 \%$ FBS. The indicated cells (300 cells per well) were seeded into of 6-well plate for $\sim 10$ 
14 days. Colonies were stained with $1 \%$ crystal violet for $10 \mathrm{~min}$ after fixation with $10 \%$ formaldehyde for $5 \mathrm{~min}$. Plating efficiency was calculated as previously described [33]. Different colony morphologies were captured under a light microscope (Olympus).

\section{Flow cytometric analysis}

Flow cytometric analyzed of apoptosis were using the FITC Annexin V Apoptosis Detection Kit I (BD, USA), and was performed previously described [34]. The cell's inner mitochondrial membrane potential $(\Delta \psi \mathrm{m})$ was detected by flow cytometric using MitoScreen JC-1 staining kit (BD), and was presented as protocol described. Briefly, cells were dissociated with trypsin and resuspended at $1 \times 10^{6}$ cells $/ \mathrm{ml}$ in Assay Buffer, and then incubated at $37{ }^{\circ} \mathrm{C}$ for $15 \mathrm{~min}$ with $10 \mu \mathrm{l} / \mathrm{ml} \mathrm{JC}-1$. Before analyzed by flow cytometer, cells were washed twice by Assay Buffer. Flow cytometry data were analyzed using FlowJo 7.6 software (TreeStar Inc., USA).

\section{Caspase- 9 or Caspase- 3 activity assays}

Activity of caspase- 9 or caspase- 3 was analysis by spectrophotometry using Caspase-9 Colorimetric Assay Kit or Caspase-3 Colorimetric Assay Kit (Keygen, China), and was presented as protocol described. Briefly, $5 \times 10^{6}$ cells or $100 \mathrm{mg}$ fresh tumor tissues were washed with cold PBS and resuspended in Lysis Buffer and incubated on ice for $30 \mathrm{~min}$. Mixed the $50 \mu \mathrm{l}$ cell suspension, $50 \mu \mathrm{l}$ Reaction Buffer, and $5 \mu$ l Caspase-3/- 9 substrate, and then incubated at $37{ }^{\circ} \mathrm{C}$ for $4 \mathrm{~h}$. The absorbance was measured at $405 \mathrm{~nm}$, and BCA protein quantitative analysis was used as the reference to normal each experiment groups.

\section{Side population analysis}

The cell suspensions were labeled with Hoechst 33,342 (Molecular probes - \#H-.

3570) dye for side population analysis as per standard protocol [35]. Briefly, cells were resuspended at $1 \times$ prewarmed OptiMEM (Gibco, USA) containing 2\% FBS (Gibco, USA) at a density of $10^{6} / \mathrm{mL}$. Hoechst 33,342 dye was added at a final concentration of $5 \mathrm{lg} / \mathrm{mL}$ in the presence or absence of verapamil (50 lmol/L; Sigma) and the cells were incubated at $37{ }^{\circ} \mathrm{C}$ for 90 min with intermittent shaking. At the end of the incubation, the cells were washed with OptiMem containing 2\% FBS and centrifuged down at $4{ }^{\circ} \mathrm{C}$, and resuspended in ice-cold OptiMem containing $2 \% \mathrm{FBS}$ and $10 \mathrm{mmol} / \mathrm{L}$ HEPES. Propidium iodide (Sigma, USA) at a final concentration of $2 \mathrm{lg} / \mathrm{mL}$ was added to the cells to gate viable cells. The cells were filtered through a 40-lm cell strainer to obtain single cell suspension before sorting. Analysis and sorting was done on a FACS Arial (Becton Dickinson). The Hoechst 33,342 dye was excited at $355 \mathrm{~nm}$ and its dual-wavelength emission at blue and red region was plotted to get the SP scatter.

\section{Spheroid formation assay}

Cells (500 cells/well) were seeded into 6-well Ultra Low Cluster plates (Corning) and cultured as previously described [36]. After 10-12 days, the number of cell spheroids (tight, spherical, non-adherent masses > $50 \mu \mathrm{m}$ in diameter) were counted, and images of the spheroids were scored under an inverse microscope (spheroids formation efficiency $=$ colonies/input cells $\times$ $100 \%)$.

\section{Tumor xenografts}

Four-week-old BALB/c-nu female mice weighing 15-20 g were maintained in a standard pathogen-free environment where the animals were housed in sterile cages under laminar flow hoods in a $20-26{ }^{\circ} \mathrm{C}$ temperature controlled room with a 12-h light/12-h dark cycle and fed autoclaved chow and water. The 6-week-old BALB/c-nu mice were randomly divided into four groups ( $n=6$ per group). Cells $\left(1 \times 10^{6}, 1 \times 10^{5} 1 \times 10^{4}\right.$ and $\left.1 \times 10^{3}\right)$ were inoculated subcutaneously together with Matrigel (final concentration of $25 \%)$ into the inguinal folds of the nude mice respectively. To study the effect of TFAP2C on chemoresistance of CRC cells, The 4-6 week-old BALB/c-nu mice were randomly divided into four groups ( $\mathrm{n}=6$ per group) and the indicated cells $\left(2 \times 10^{6}\right)$ were inoculated subcutaneously into the inguinal folds of the nude mice. After seven days for cells inoculation, the mice were injected intraperitoneally $50 \mathrm{mg} /$ kg.d 5-FU for 4 weeks. Tumor volume was determined using an external caliper and calculated using the eq. $(\mathrm{L} \times$ W2)/2. On day 38, animals were euthanized, tumors were excised, weighed and stored in liquid nitrogen tanks.

\section{Immunohistochemistry}

The immunohistochemistry procedure and scoring of TFAP2C expression levels were performed as previously described [37]. Scores given by two independent investigators were averaged for further comparative evaluation of TFAP2C expression. The proportion of tumor cells was scored as follows: 0 (no positive tumor cells); 1 ( $<10 \%$ positive tumor cells); 2 (10-35\% positive tumor cells); 3 (35$70 \%$ positive tumor cells) and 4 ( $>70 \%$ positive tumor cells). The staining intensity was graded according to the following criteria: 0 (no staining); 1 (weak staining, light yellow); 2 (moderate staining, yellow brown) and 3 (strong staining, brown). The staining index (SI) was calculated as the product of the staining intensity score and the proportion of positive tumor cells. Using this method of assessment, we evaluated TFAP2C expression in CRC samples by determining SI, with scores of $0,1,2,3,4,6,8,9$ or 12 . An SI score 4 was the median SI of all sample tissues. High and low TFAP2C expression was stratified by the follow 
criteria: $\mathrm{SI} \leq 4$ was used to define tumors with low TFAP2C expression; SI $\geq 6$ was used to define tumors with high TFAP2C expression.

\section{Luciferase assay}

Cells $\left(4 \times 10^{4}\right)$ were seeded in triplicate in 24-well plates and cultured for $24 \mathrm{~h}$, and the luciferase reporter assay was performed as previously described [38]. Cells were transfected with $100 \mathrm{ng}$ pROCK1 or ROCK2 promoter reporter luciferase plasmid, plus 5 ng pRL-TK Renilla plasmid (Promega) using Lipofectamine 3000 (Invitrogen) according to the manufacturer's recommendation. Luciferase and Renilla signals were measured $36 \mathrm{~h}$ after transfection using a Dual Luciferase Reporter Assay Kit (Promega) according to the manufacturer's protocol.

\section{Immunofluorescence}

Cells were seeded on glass culture slides (BD Biosciences) and fixed with $4 \%$ cold methanol at $-20{ }^{\circ} \mathrm{C}$ for $10 \mathrm{~min}$. Subsequently, cells were blocked with $10 \%$ goat serum for $1 \mathrm{~h}$ and incubated with primary antibodies against YAP (Cell Signaling Technology, Cat. 14,074), TAZ (Abcam, Cat. ab224239) or Phalloidin-iFluor 488 Reagent - CytoPainter (Abcam, Cat. ab176753) at room temperature for $2 \mathrm{~h}$ and then incubated with secondary antibody FITC for $1 \mathrm{~h}$ at room temperature. After counterstained with DAPI (Invitrogen), the slide was observed under a confocal microscope (Zeiss).

\section{Statistical analysis}

All values are presented as means \pm standard deviation (SD). Significant differences were determined using GraphPad 5.0 software (USA). Student's t-test was used to determine statistical differences between two groups. One-way ANOVA was used to determine statistical differences between multiple testing. The chi-square test was used to analyze the relationship between TFAP2C expression and clinicopathological characteristics. Survival curves were plotted using the Kaplan Meier method and compared by log-rank test. $P<0.05$ was considered significant. All the experiments were repeated three times.

\section{Results}

TFAP2C is upregulated in colorectal cancer cell lines and tissues

Through analyzing several colorectal cancer RNA sequencing datasets from The Cancer Genome Atlas (TCGA) and ArrayExpress, we found that TFAP2C expression was dramatically elevated in CRC tissues compared with the adjacent normal colorectal tissues (Fig. 1a and Additional file 5: Figure S1). The TFAP2C expression level in 50 paired primary CRC tissue samples from TCGA was further analyzed and the result showed that TFAP2C expression in the majority of CRC tissues was higher than their matched adjacent normal tissue samples (Fig. 1b). We further validated TFAP2C expression in our own 8 paired colorectal cancer tissues and found that mRNA and protein expression elevels of TFAP2C was differentially upregulated in $\mathrm{CRC}$ tissue samples compared with the matched adjacent normal tissue samples (Fig. 1c and d). Consistently, expression levels of TFAP2C were upregulated in CRC cells compared with normal colon epithelial cell CMEC, except RKO cell (Fig. 1e and f). Thus, these results indicate that overexpression of TFAP2C contributes to the progression of colorectal cancer.

\section{Overexpression of TFAP2C correlates with advanced clinico0pathological features and poor prognosis in CRC patients}

We further examined TFAP2C expression by immunohistochemical analysis of 378 human CRC tissues (Additional file 2: Table S2) and found TFAP2C expression was primarily detected within the cytoplasm and nucleus, and the expression levels of TFAP2C positively correlated with clinical stages (Fig. 2a). High expression of TFAP2C was observed in 188/378 CRC tissues (49.7\%) (Fig. 2b). In addition, there were potent positive associations between TFAP2C expression and T classification, $\mathrm{N}$ classification, $\mathrm{M}$ classification and clinical stage (Fig. 2c-f and Additional file 6: Table S5). Kaplan-Meier survival analysis indicated that CRC patients with high TFAP2C expression exhibited shorter overall survivals and progression-free survivals (Fig. $2 g$ and h), which were further verified by the analysis results from several publicly available CRC dataset (Additional file 7: Figure S2A-F). Collectively, these results demonstrate that the overexpression of TFAP2C correlates with advanced clinicopathological characteristics, and poor prognosis and disease progression in CRC patients.

\section{Overexpression of TFAP2C confers chemotherapeutic resistance in CRC cells}

Interestingly, we found that high expression of TFAP2C positively with poor chemotherapy response in CRC patients via analyzing CRC datasets from TCGA and GSE28702 (Additional file 8: Figure S3A and B), which was further validated in our CRC tissues (Additional file 8: Figure S3C). We further analyzed the correlation of TFAP2C expression with chemotherapeutic response in CRC cells via calculating the apoptotic ratio of CRC cells under treatment of 5-FU, and found that CRC cells with high expression level of TFAP2C displayed more resistance to 5-FU (Additional file 8: Figure S3D). Furthermore, negative correlation of TFAP2C expression with apoptotic ration was found in CRC cells (Additional file 8: Figure S3E and F). These finding suggest that high expression level of TFAP2C may be associated with chemoresistance in CRC. 

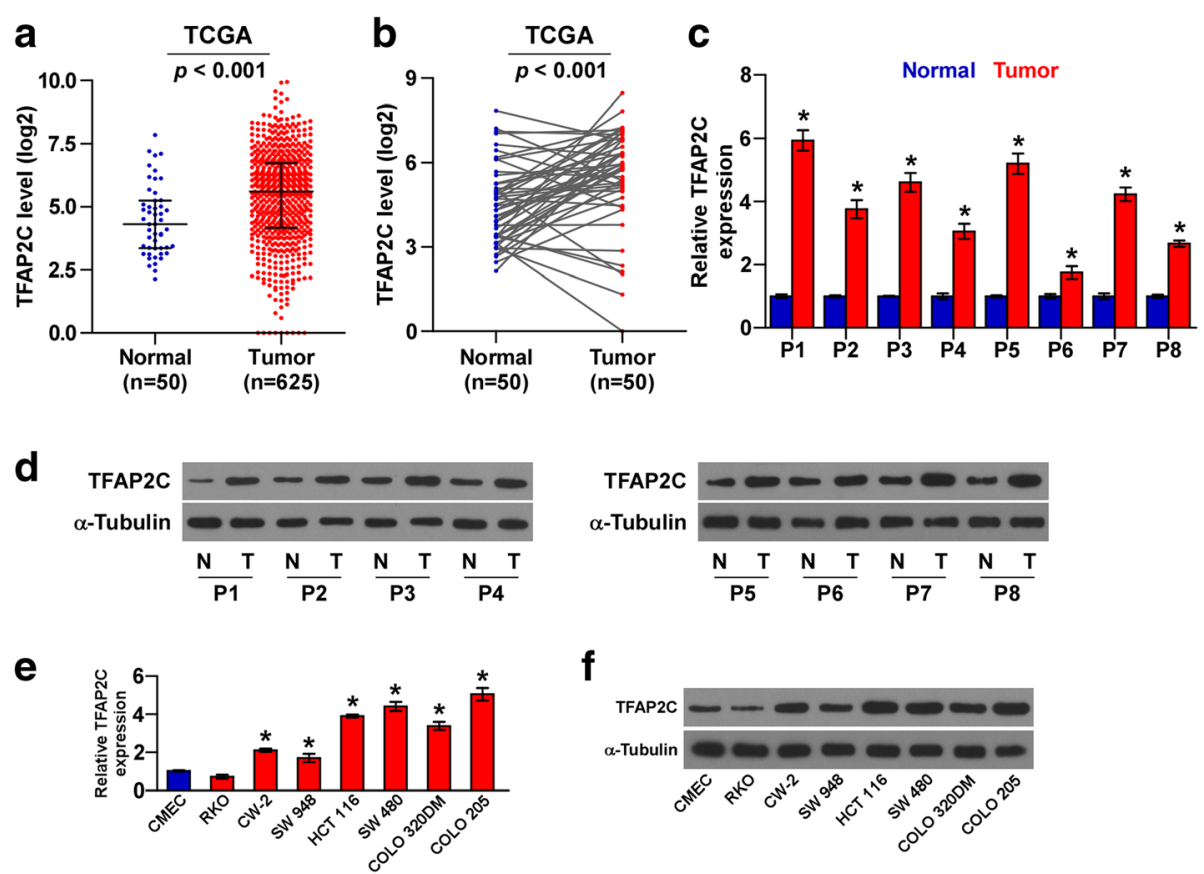

f

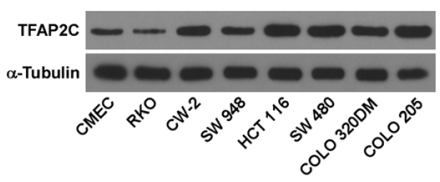

Fig. 1 TFAP2C is upregulated in CRC tissues and cells. a TFAP2C expression level was elevated in colorectal cancer tissues compared with the adjacent normal tissues as assessed by analyzing the TCGA colorectal cancer RNA sequencing dataset (Adjacent normal tissue, $n=50 ;$ Colorectal cancer, $n=625$ ). $\mathbf{b}$ TFAP2C expression level was elevated in 50 paired colorectal cancer tissues compared with the adjacent normal tissues as assessed by analyzing the TCGA colorectal cancer RNA sequencing dataset. $\mathbf{c}$ and $\mathbf{d}$ Real-time PCR analysis and Western blotting of TFAP2C expression in 8 paired CRC tissues. a-Tubulin was detected as a loading control in the Western blot and GAPDH was used as endogenous controls in RT-PCR. Each bar represents the mean values \pm SD of three independent experiments. ${ }^{*} P<0.05$. e and $\mathbf{f}$ Real-time PCR and Western blotting analysis of TFAP2C expression in one normal colon epithelial cell CMEC and 7 CRC cell lines. GAPDH was used as endogenous controls in RT-PCR and a-Tubulin was detected as a loading control in the Western blot. Each bar represents the mean values \pm SD of three independent experiments. ${ }^{*} P<0.05$

To determine the stimulatory roles of TFAP2C in the chemotherapeutic resistance of $\mathrm{CRC}$, we first constructed TFAP2C-stably expressing SW480 and HCT116 CRC cell lines by ectopically overexpressing TFAP2C and endogenously knocking down TFAP2C via retrovirus infection (Additional file 9: Figure S4A and B). As shown in Fig. 3a, upregulating TFAP2C reduced, while silencing TFAP2C increased the apoptosis rate of CRC cells treated with 5-FU. Moreover, TFAP2C overexpression enhanced, while silencing TFAP2C decreased, the mitochondrial potential of CRC cells under treatment of 5-FU (Fig. 3b). We further examined the effect of TFAP2C on the expression levels of the anti-apoptotic proteins BCL2 and BCL2L1 and found upregulating TFAP2C increased, while silencing TFAP2C reduced BCL2 and BCL2L1 expression (Fig. 3c). The effect of TFAP2C on the activity of caspase- 3 or -9 was further investigated and the results revealed that upregulating TFAP2C repressed the activity of caspase- 3 or -9 ; conversely, silencing TFAP2C increased the activity of caspase-3 or -9 (Fig. 3d and e). Furthermore, our results demonstrated that silencing TFAP2C dramatically reduced the cell proliferation ability via CCK- 8 , colony formation and anchorage-independent growth assays; however, upregulating TFAP2C had no obvious effect on the proliferation ability of CRC cells (Additional file 9: Figure S4C-E), suggesting that overexpression of TFAP2C promotes chemoresistance of CRC cells to 5-FU independent on the effects of TFAP2C on cell proliferation. Collectively, these results indicate that TFAP2C promotes chemoresistance of CRC cells to 5 -FU in vitro.

\section{Silencing TFAP2C restores chemosensitivity of CRC cells to 5 -FU in vivo}

We further examined the effect of TFAP2C on the chemoresistance of CRC cells in vivo. Mice were randomly divided into five groups $(n=6 /$ group $)$ and $2 \times 10^{6}$ HCT116 cells of control, TFAP2C-overexpression, vector, TFAP2CRNAi\#1 and TFAP2C-RNAi\#2 were inoculated subcutaneously in the left dorsal flank respectively. A weeks later, each group of mice were injected intraperitoneally with 5FU (50 mg/kg.d). As shown in Fig. 4a-c, the tumor volumes and weight were increased in the TFAP2C -overexpressing plus 5-FU mice group, but were dramatically decreased in the TFAP2C-silencing plus 5-FU mice group, compared to the corresponding mice controls. Furthermore, the activity of caspase- 3 or -9 in the tumor tissues from TFAP2C -overexpressing plus 5-FU mice group was robustly suppressed, but enhanced in the tumor tissues from TFAP2C 


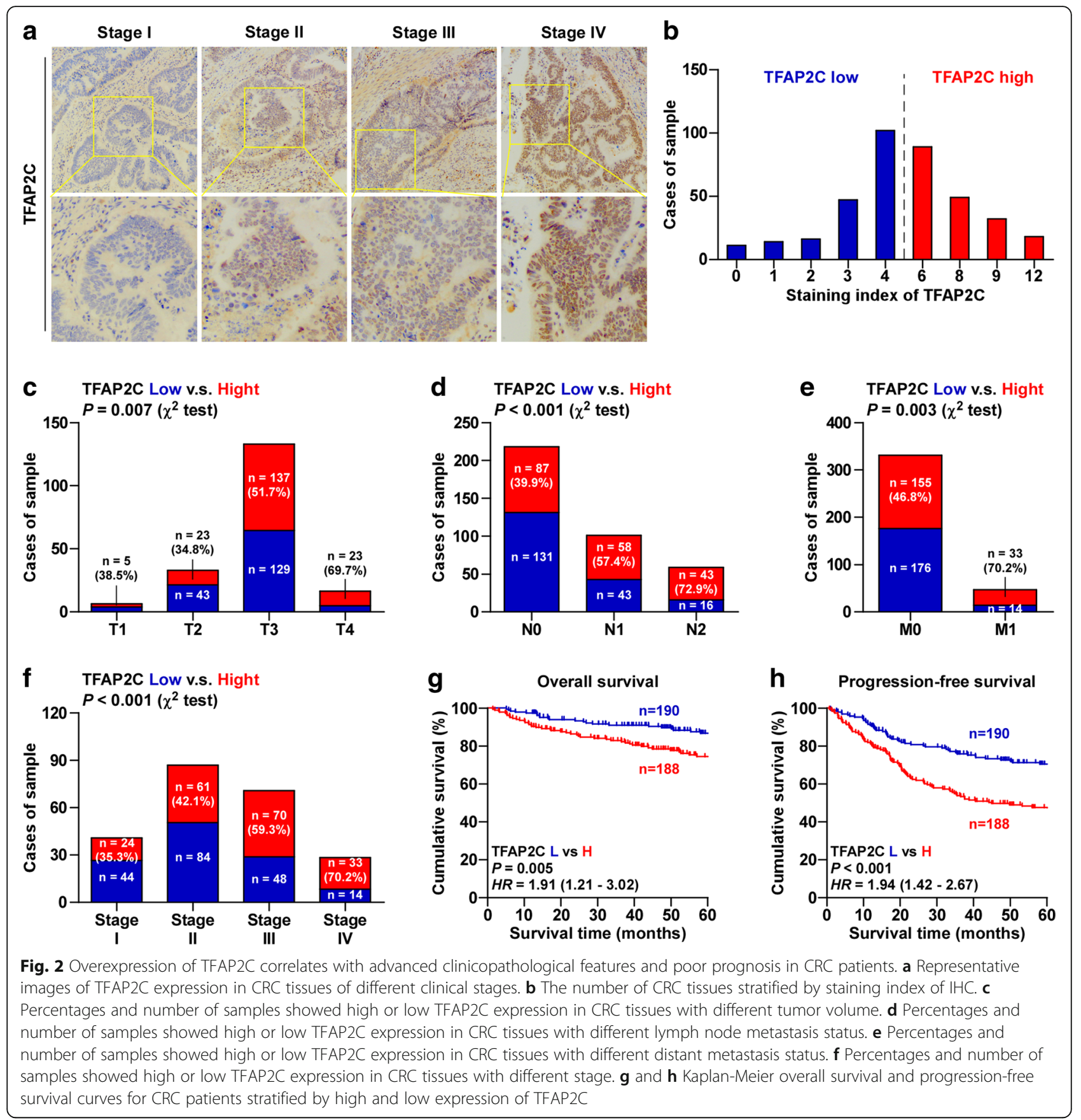

-silencing plus 5-FU mice group (Fig. 4d). Collectively, these findings indicate that silencing TFAP2C improves chemotherapeutic sensitivity of CRC cells to 5-FU in vivo.

TFAP2C promotes cancer stem characteristics in CRC cells Several lines of evidence have shown that cancer stem cells (CSCs) are crucial for the induction and maintenance of chemotherapeutic resistance in cancers $[8,9]$, which is responsible for the failure of chemotherapy in cancer treatment, suggesting that these two cellular processes are intimately linked. Therefore, we further evaluated the effects of TFAP2C on CSCs characteristics in CRC cells. Spheroids formation assay was performed and the results showed that overexpression of TFAP2C increased spheroids formation ability of CRC cells, while silencing TFAP2C decreased spheroids formation ability (Fig. 5a). Side population (SP) analysis further revealed that upregulating TFAP2C enhanced, while silencing TFAP2C decreased the fraction of SP cells (Fig. 5b). The effects of TFAP2C on expression levels of stem cell factors, including NANOG, BMI-1, OCT4 and SOX2, were further examined, and the results showed that upregulating 


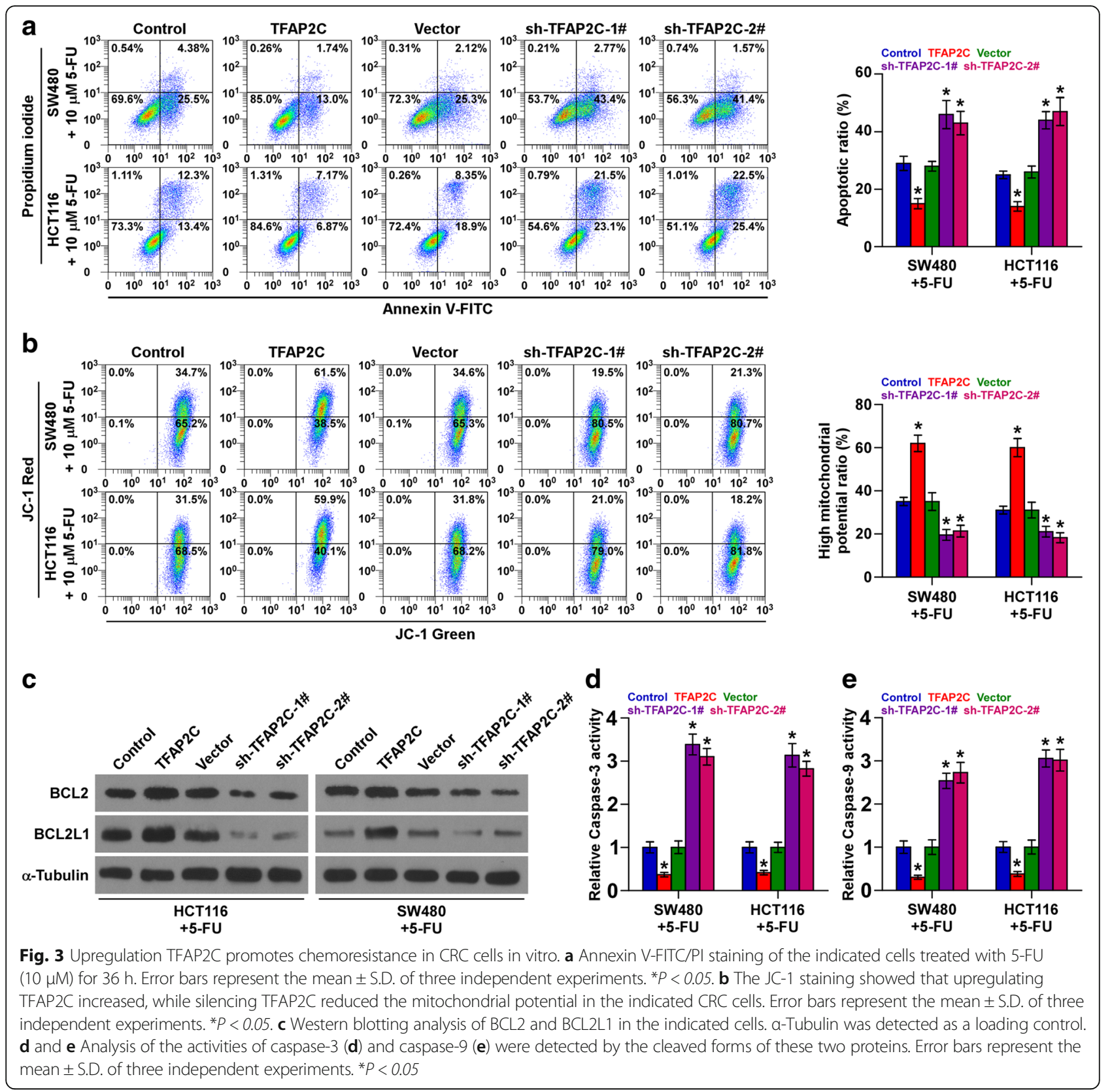

TFAP2C enhanced, while silencing TFAP2C reduced the expression of these stem cell factors (Additional file 10: Figure S5A and B).

The effect of TFAP2C on tumorigenesis of CRC cells was further investigated in vivo. As shown in Fig. 5c, the number of tumor initiating cells (TIC) required to develop tumor in mice were significantly reduced in the TFAP2C-overexpressing mice group, but enhanced in TFAP2C-silenced mice group. Furthermore, the tumors formed by TFAP2C-overexpressing cells were larger than tumors in the control group after implantation of $1 \times 10^{6}, 1 \times 10^{5}$ or $1 \times 10^{4}$ cells; conversely, tumors weight in the TFAP2C-silencing group were lower than the tumors in the control group (Fig. 5d). Importantly, tumors were only detected after inoculation of $1 \times 10^{3}$ TFAP2C-overexpressing cells (Fig. 5d and Additional file 10: Figure S5C). Collectively, these findings indicate that TFAP2C promotes CSCs characteristics of CRC cells in vitro and in vivo.

TFAP2C inactivates hippo signaling pathway in CRC cells To determine the underlying signaling pathways mediating the stimulatory effects of TFAP2C on chemoresistance and stemness in CRC cells, luciferase reporter plasmids of multiple signaling pathways were transfected into CRC cells respectively, and we found that 

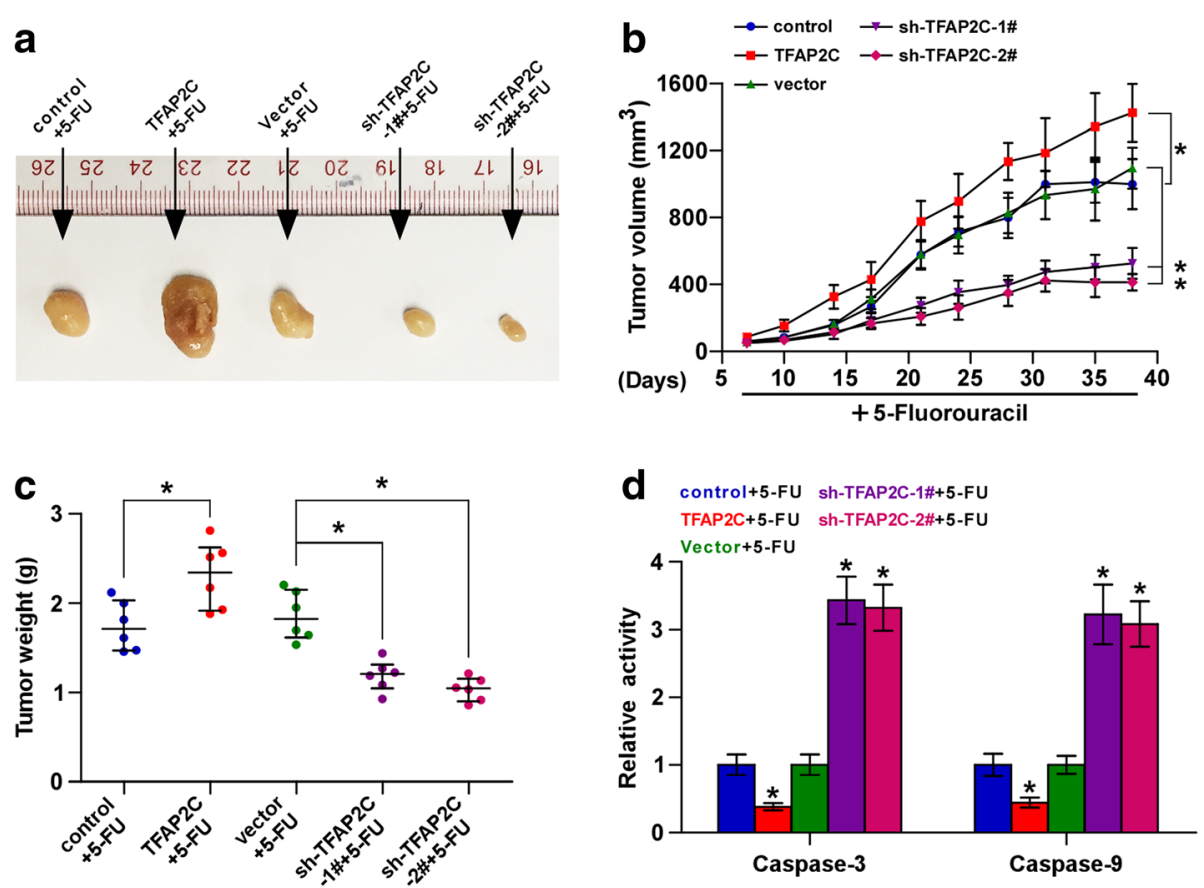

Fig. 4 Downregulation of TFAP2C improves chemosensitivity of CRC cells to 5-FU in vivo. a Representative images of the tumors are shown in the xenograft model of nude mice. b After one weeks of inoculating HCT116 cells, mice were intraperitoneal injected with 50 mg/kg 5-FU per day for four weeks. Tumor volumes in the indicated groups were measured from the fifth day at five days interval. Data presented are the mean \pm s.d. c Tumor weights of each group. $\mathbf{d}$ Analysis of the activities of caspase- 3 and caspase- 9 were examined in the indicated tumor from mice. Error bars represent the mean \pm S.D. of three independent experiments. ${ }^{*} P<0.05$

transcriptional activity of TEAD, the transcriptional coactivators of Hippo signaling pathway, was the most highly regulated by upregulation of TFAP2C, but silenced by downregulation of TFAP2C in CRC cells (Additional file 11: Figure S6A). GSEA was further performed and the results showed that TFAP2C expression level was positively associated with the YAP and TAZactivated gene signatures, suggesting that the inactivation of Hippo signaling pathway may be associated with the pro-tumor effects of TFAP2C (Additional file 11: Figure S6B and C). Analysis of CRC datasets from TCGA revealed that expression levels of TFAP2C positively correlated with the protein expression levels of transcriptional co-activators YAP and TAZ of Hippo signaling (Additional file 11: Figure S6D-G). Immunofluorescence staining demonstrated that upregulating TFAP2C enhanced, while silencing TFAP2C reduced the nuclear translocation of YAP and TAZ (Fig. 6a-c). Luciferase assay showed that upregulating TFAP2C significantly enhanced, while silencing TFAP2C repressed, TEAD-dependent luciferase activity in CRC cells (Fig. 6d). Moreover, cellular fractionation and western blotting analysis revealed that overexpression of TFAP2C reduced the p-MST1/2, pLATS1 and p-YAP levels, increased cytoplasmic and nuclear YAP and TAZ levels, and had no effect on total expression level of MST1/2 and LATS1 in CRC cells; however, silencing TFAP2C yielded an opposite effects (Fig. 6e and f). Real-time PCR analysis showed that upregulation of TFAP2C increased, while silencing TFAP2C decreased expression levels of multiple downstream genes, including CTGF, CYR61, HOXA1 and SOX9 in CRC cells, but had no effect on expression levels of house-keeping genes RPL13A and PPIA (Fig. 6g). Thus, these results demonstrate that TFAP2C inactivates Hippo signaling pathway in CRC cells.

\section{TFAP2C promotes chemoresistance and stemness via inactivating hippo signaling}

We further determine whether activity of Hippo signaling is involved in the effects of TFAP2C on chemoresistance and stemness in CRC cells, and found that individual silencing of YAP or TAZ attenuated the stimulatory effects of TFAP2C overexpression on sphere formation, SP fraction and mitochondrial potential in CRC cells (Additional file 12: Figure S7A-C). Furthermore, apoptotic ratio of CRC cells repressed by TFAP2C overexpression was dramatically reversed by silencing YAP or TAZ (Additional file 12: Figure S7D). Taken together, these findings indicate that TFAP2C promotes the chemoresistance and stemness via inactivating Hippo signaling in CRC cells. 


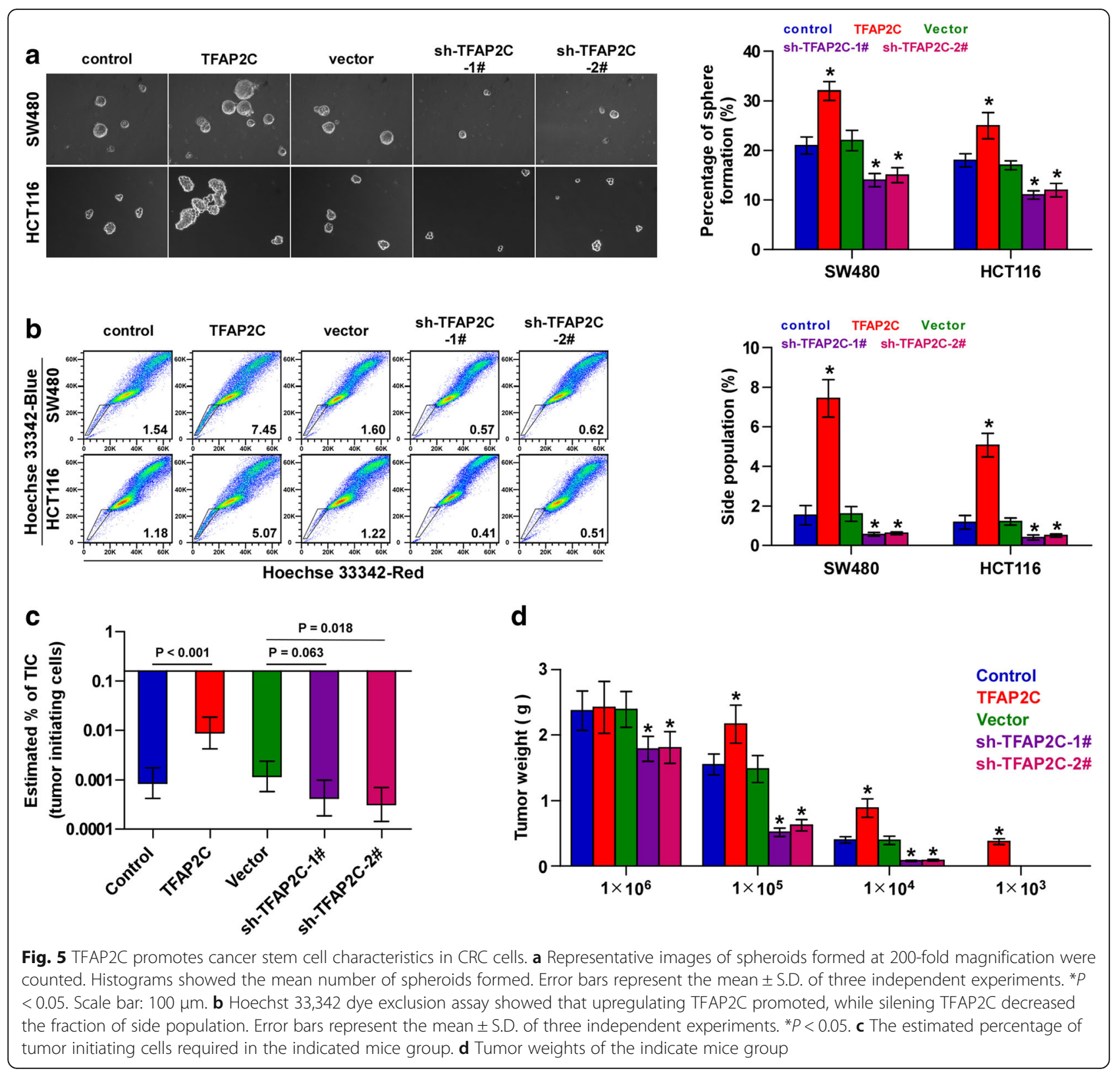

TFAP2C promotes stemness and chemoresistance of CRC cells via transcriptionally activating ROCK1 and ROCK2

Through analyzing JASPAR, we found several TFAP2Cbinding motifs inside the putative promoter region of ROCK1 and ROCK2 (Additional file 13: Figure S8A and B), both of which have been reported to be negative regulators of Hippo signaling pathway via inhibition of LATS activity [39]. The UCSC bioinformatics identified four potential binding sites of TFAP2C in the promoter region of ROCK1 and ROCK2 (Additional file 13: Figure S8C and D). RTPCR and Western blot analysis showed that upregulation of TFAP2C enhanced, while silencing TFAP2C reduced mRNA and protein expression levels of ROCK1 and ROCK2 (Fig. 7a-c). A ChIP assay indicated that TFAP2C could bind to the P2 and P3 binding sites in the promoter region of ROCK1 and P1 and P2 binding sites in ROCK2 in CRC cells (Fig. 7d and e, and Additional file 14: Figure S7A and B). Furthermore, an enhancement of the ROCK1 and ROCK 2 promoter luciferase activity was observed on upregulation of TFAP2C in CRC cells; conversely, downregulation of TFAP2C significantly reduced the luciferase activity (Fig. 7f and g, and Additional file 14: Figure S7C and D). However, upregulation or downregulation of TFAP2C had no effect on the luciferase activity of the ROCK1 and ROCK2 promoter that contained all mutations in the P2 and P3 binding sites (Fig. 7a, b, g and h, and Additional file 12: Figure S7E and F). Importantly, Y-27632, a specific inhibitor of ROCK1 and ROCK2, significantly 

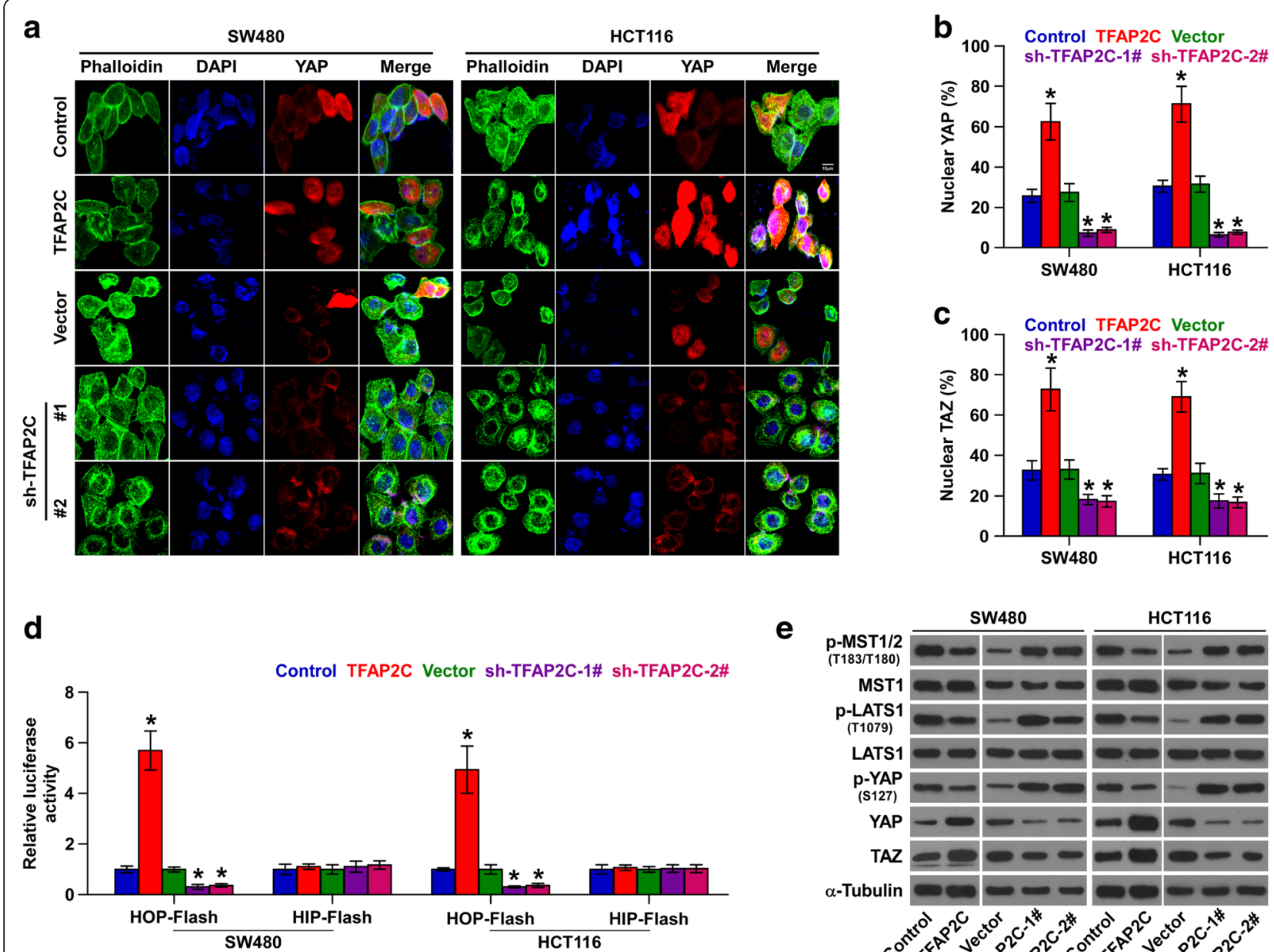

e

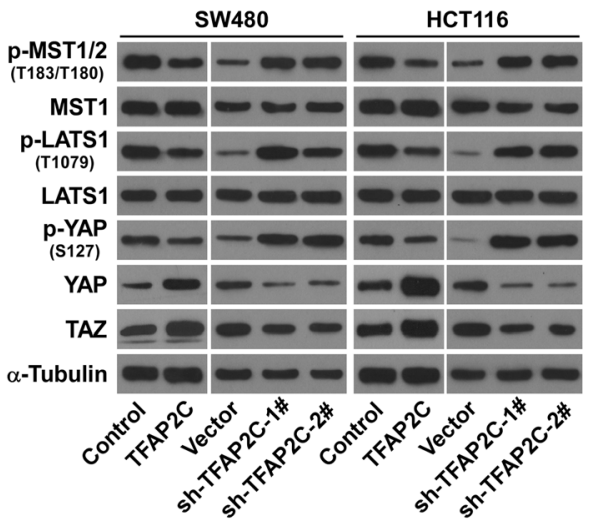

$\mathbf{f}$

g
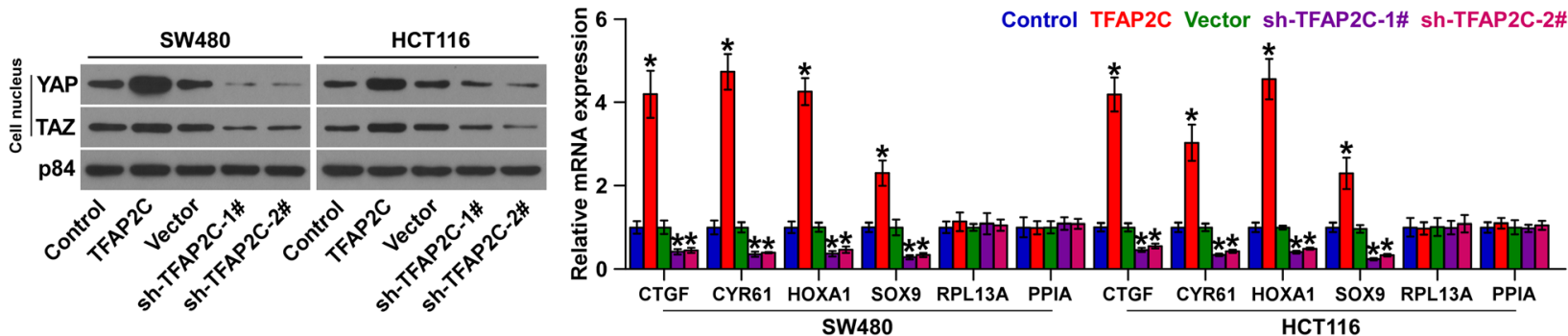

Fig. 6 TFAP2C inactivates Hippo signaling pathway in CRC cells. a Representative immunofluorescent images of CRC cells were immunostained with YAP antibody (red) or phalloidin (green) in the indicated CRC cells. b and $\mathbf{c}$ The percentage of nuclear YAP+ (b) and nuclear TAZ+ (c) cell number via immunostaining in the indicated groups. ${ }^{*} P<0.05$. $\mathbf{d}$ TEAD transcriptional activity was assessed by HOP-Flash luciferase reporter in the indicated cells. Error bars represent the mean \pm S.D. of three independent experiments. ${ }^{*} P<0.05$. e Western blotting of $p-M S T 1 / 2, M S T 1 / 2, p-L A T S 1$, LAST1, p-YAP, YAP and TAZ expression in the indicated cells. a-tubulin was used as the loading control. $\mathbf{f}$ Cellular fractionation and western blotting analysis of nuclear YAP and TAZ expression in the indicated cells. The nuclear protein p84 was used as the nuclear protein marker. $\mathbf{g}$ Real-time PCR analysis of CTGF, CYR61, HOXA1, SOX9, RPL13A and PPIA in the indicated cells. Error bars represent the mean \pm S.D. of three independent experiments. ${ }^{*} P<0.05$

repressed stemness and chemoresistance in TFAP2Coverexpressing cells (Additional file 15: Figure S10A-D), as well as increased levels of p-MST1/2, p-LATS1 and p-
YAP and decreased total and nuclear levels of YAP and TAZ (Additional file 15: Figure S10E-I). Collectively, our results indicate that TFAP2C promotes stemness and 


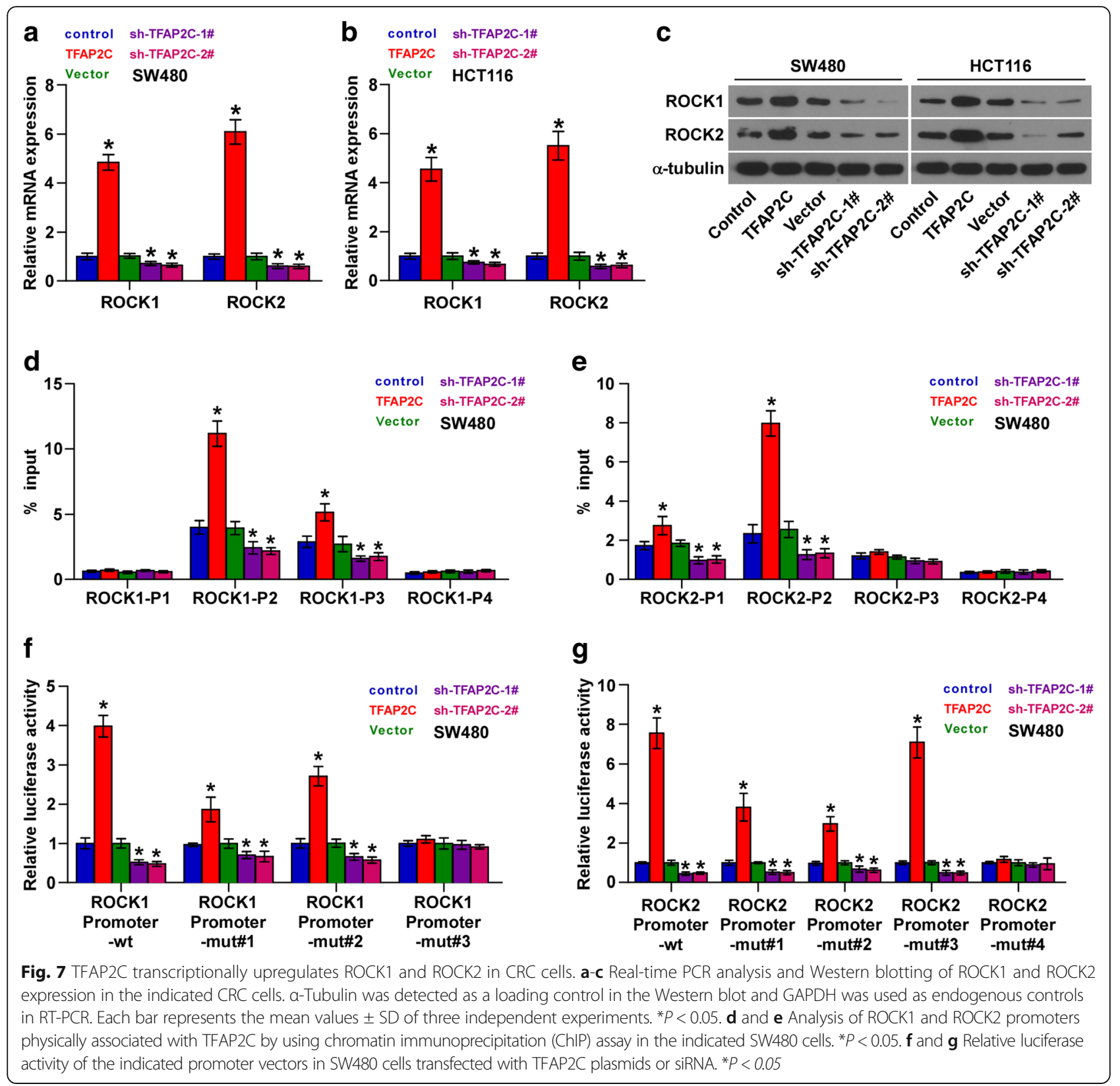

chemotherapeutic resistance of CRC cells via transcriptionally activating ROCK1 and ROCK2, leading to inactivation of Hippo signaling pathway in CRC cells.

Clinical relation of TFAP2C with hippo signaling activity in human CRC tissues

To determine the clinical correlation of TFAP2C with Hippo signaling activity in clinical CRC tissues, the protein levels of TFAP2C, p-MST1/2, p-LATS1, YAP and TAZ expression were examined in four 5-FU resistant and four 5-FU sensitive CRC tissues. As shown in Additional file 16: Figure S11A-F, TFAP2C, YAP and TAZ expression levels were upregulated in resistant CRC tissues (T1-4) compared with that in sensitive CRC tissues (T5-8); conversely, p-MST1/2 and p-LATS1 expression were reduced in resistant CRC tissues. Thus, our results indicate that there is a positive clinical correlation of TFAP2C with inactivation of Hippo signaling in CRC tissues.

\section{Discussion}

In the current study, we found that TFAP2C was upregulated in CRC tissues and cells, which correlated with advanced clinicopathological features, poor prognosis and disease progression in CRC patients. Furthermore, upregulation of TFAP2C enhanced, while silencing 
TFAP2C attenuated stemness and chemotherapeutic resistance in CRC cells in vitro and in vivo. Our results further reveal that TFAP2C promoted CSCs characteristics and chemoresistance via transcriptionally upregulating $\mathrm{ROKC} 1$ and ROCK2 expression, leading to inactivation of Hippo signaling. Therefore, our findings uncover a novel mechanism by which TFAP2C promotes CSCs characteristics and chemoresistance in CRC.

Accumulating studies have shown that aberrant expression of AP-2 proteins was implicated in the development, progression and metastasis in several human cancers. For example, TFAP2C expression was elevated in lung carcinoma and high expression of TFAP2C promoted cell cycle activation and lung carcinoma cell tumorigenesis via by upregulating the oncogenic miR-183 and downregulating tumor-suppressive miRNA-33a [40]. Moreover, low TFAP2B expression was reported in primary neuroblastomas, which correlated with poor prognosis via promoting proliferation and cell cycle progression [41]. Furthermore, different members of AP-2 proteins play opposite roles in the same tumor type. In breast cancer, TFAP2A functioned as a tumor suppressor; conversely, TFAP2C played an oncogenic role in the development and progression of breast cancer [42]. These studies indicated that although belonged to the AP-2 family, different members of AP-2 proteins function as either oncogene or tumor suppressor in cancers. In the current study, we found that TFAP2C was remarkably elevated in CRC tissues and high expression of TFAP2C correlated with advanced clinicopathological features, poor prognosis and disease progression in CRC patients. Furthermore, upregulation of TFAP2C enhanced the chemotherapeutic resistance in CRC cells; conversely silencing TFAP2C yielded an opposite effect. Therefore, our findings indicate that TFAP2C plays an oncogenic role in CRC via promoting chemoresistance and stemness of CRC cells.

TFAP2C has been reported to be upregulated in various types of cancer, including lung carcinoma, breast cancer, and high expression of TFAP2C significantly correlated with poor prognosis via promoting the growth and proliferation $[40,43,44]$. However, other studies have shown that TFAP2C played a tumor suppressive role in several human cancers, such as melanoma, endometrial cancer $[45,46]$. Interestingly, Bogachek and colleagues reported that TFAP2C regulated multiple breast cancer-related genes, and loss of TFAP2C induced epithelial-mesenchymal transition in breast cancer cells [47]. These studies suggest that the pro- and anti-tumor roles of TFAP2C are function and tumor type dependent. However, the clinical significance and biological role of TFAP2C in colorectal cancer remain largely unknown. In this study, high expression of TFAP2C was observed in CRC tissues, which correlated with advanced clinicopathological features, poor prognosis and disease progression in CRC patients. Moreover, our results demonstrated that TFAP2C promoted the chemoresistance and stemness of CRC cells in vitro and in vivo, further determining the tumor-stimulatory role of TFAP2C in CRC.

Numerous studies have reported that several regulatory mechanisms were responsible for the inactivation of Hippo signaling, which played an important role in the chemotherapeutic resistance of cancer. Studies have consistently shown that inactivation of Hippo signaling by downregulation of the Hippo pathway components mammalian MST1/2 andLATS1/2 contributed to resistance of cancer cells to chemotherapeutic drugs [48-50]. Furthermore, upregulation of YAP or TAZ conferred chemotherapeutic resistance in multiple cancer types [51-53]. Recently, it was reported that Rho-associated protein kinase (ROCK) repressed activity of Hippo signaling through inhibition of LATS activity and cell polarity in the outside cells [39]. Importantly, Cao and colleagues reported that TFAP2C inhibited Hippo signaling dependent on ROCK activity [54]. It remains unclear, however, how ROCK mediates the inhibitory effect of TFAP2C on Hippo signaling activity and whether TFAP2C/ROCK/Hippo signaling promotes chemotherapeutic resistance in CRC. In this study, our results revealed that TFAP2C transcriptionally activated ROCK1 and ROCK2 via binding to the promoter region of ROCK1 and ROCK2 in CRC cells. Importantly, the stimulatory effects of TFAP2C on chemoresistance and stemness in CRC cells were effectively attenuated by the specific inhibitor of ROCK1 and ROCK2, Y-27632. Thus, our results uncover a novel mechanism by which TFAP2C promotes chemotherapeutic resistance and stemness in CRC cells.

It has been widely documented that AP- 2 members may be used as a prognostic marker in numerous human tumor types. A study by Ikram et al. showed that low TFAP2B expression caused by CpG methylation of the TFAP2B locus in primary neuroblastomas significantly promoted proliferation and cell cycle progression and low expression of TFAP2B significantly associated with unfavorable prognostic markers as well as adverse patient outcome [41]. Conversely, TFAP2A and TFAP2B correlated with good overall and disease-free survival in breast cancer patients [55]. These studies indicated that different AP-2 proteins predict different prognosis in different tumor types. Furthermore, TFAP2C has been extensively reported to be a poor prognostic marker in several human cancers, including breast cancer, lung carcinoma, [16, 40,44]. However, the correlation of TFAP2C with prognosis of CRC patients remains unknown. In this study, our results found that TFAP2C expression level was increased in CRC tissues and high expression of TFAP2C correlated with advanced clinicopathological features. More importantly, Kaplan-Meier survival analysis revealed that CRC patients with high TFAP2C expression exhibited shorter overall survivals and progression-free survivals. Thus, these findings 
identify TFAP2C as a potential prognostic marker in CRC patients.

\section{Conclusion}

In summary, our findings demonstrate that TFAP2C inactivates Hippo signaling via transcriptionally upregulating ROCK1 and ROCK2 expression, which further promotes chemotherapeutic resistance and stemness in CRC. Therefore, better understanding the underlying mechanism and the specific role of TFAP2C in the pathogenesis of CRC facilitates the development of novel therapeutic strategies for treatment of CRC.

\section{Additional files}

Additional file 1: Table S1. The basic information of 8 colorectal cancer patients for TFAP2C mRNA and protein expression analysis. (PDF $51 \mathrm{~kb}$ )

Additional file 2: Table S2. The basic information of 378 patients with colorectal cancer for TFAP2C immunohistochemical staining analysis. (PDF $55 \mathrm{~kb}$ )

Additional file 3: Table S3. A list of primers used in the reactions for real-time RT-PCR. (PDF $61 \mathrm{~kb}$ )

Additional file 4: Table S4. A list of primers used in the reactions for clone PCR. (PDF $49 \mathrm{~kb}$ )

Additional file 5: Table S5. The relationship between TFAP2C IHC expression level and clinical. (PDF $61 \mathrm{~kb}$ )

Additional file 6: Figure S1. TFAP2C expression level was elevated in colorectal cancer tissues compared with the benign colorectal lesions as assessed by analyzing the GSE17538 colorectal cancer RNA sequencing dataset (Benign, $n=6$; Colorectal cancer, $n=232$ ). (PDF $35 \mathrm{~kb}$ )

Additional file 7: Figure S2. Overexpression of TFAP2C is associated poor overall and progression-free survivals in CRC patients (A-C) Overall survival curves from the TCGA, GSE17538 and GSE38832 profiles for CRC patients stratified by high and low expression of TFAP2C. (D-F)

Progression-free survival curves from the TCGA, GSE17538 and GSE38832 profiles for CRC patients stratified by high and low expression of TFAP2C. (PDF $233 \mathrm{~kb}$ )

Additional file 8: Figure S3. Overexpression of TFAP2C is associated with poor chemotherapy response. (A and B) TFAP2C expression levels were much higher in CRC patients with poor chemotherapy response as assessed by analyzing the TCGA and GSE28702 CRC RNA sequencing datasets. (C) Percentages and number of samples showed high or low TFAP2C expression in CRC patients with different chemotherapy response in our CRC tissues. (D) Apoptotic ratio of CRC cells under treatment of 5-

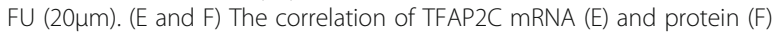
expression levels with apoptotic ratio in CRC cells after treated with 20um 5-FU. (PDF $166 \mathrm{~kb}$ )

Additional file 9: Figure S4. Silencing TFAP2C inhibits proliferation ability of CRC cells. (A and B) Real-time PCR and Western blot of the indicated CRC cells transfected with TFAP2C -vector, TFAP2C, TFAP2C -RNAivector, TFAP2C -RNAi\#1 and TFAP2C -RNAi\#2. GAPDH was used as endogenous controls in RT-PCR and a-Tubulin was detected as a loading control in the Western blot. Each bar represents the mean values \pm SD of three independent experiments. ${ }^{*} P<0.05$. (C) CCK-8 assay revealed that silencing TFAP2C decreased the proliferation rate in CRC cells. Each bar represents the mean values \pm SD of three independent experiments. ${ }^{*} P<$ 0.05. (D) downregulation of endogenous TFAP2C reduced, the mean colony number in the colony formation assay. Each bar represents the mean values \pm SD of three independent experiments. ${ }^{*} P<0.05$. (E) Representative micrographs and colony numbers in the indicated group in the anchorage-independent growth assay. Each bar represents the mean values \pm SD of three independent experiments. $* P<0.05$. (PDF $167 \mathrm{~kb}$ )
Additional file 10: Figure S5. (A and B) Real-time PCR analysis of OCT4A, SOX2, NANOG and BMI-1 expression in the indicated cells. GAPDH was used as the loading control. Error bars represent the mean \pm S.D. of three independent experiments. ${ }^{*} P<0.05$. (C) The formation number of tumor initiated by different amounts of HCT116 cells in nude mice. (PDF 106 kb)

Additional file 11: Figure S6. (A) Activity of luciferase reporter constructs of several signaling pathway were examined in the TFAP2Coverexpressing or -silencing CRC cells. ( $B$ and C) TFAP2C expression level was positively associated with the YAP and TAZ-activated gene signatures. (D-G) TFAP2C expression level is positively associated with the protein expression levels of transcriptional co-activators YAP and TAZ of Hippo signaling pathway as assessed through CRC dataset from TCGA. (PDF 162 kb)

Additional file 12: Figure S7. ( $A$ and $B$ ) Individual silencing of YAP or TAZ attenuated the sphere formation ability and SP fraction in the TFAP2C-overexpressing CRC cells. ${ }^{*} P<0.05$. (C and D) Individual silencing of YAP or TAZ reversed the effects of TFAP2C upregulation on mitochondrial potential and apoptotic ratio in CRC cells. ${ }^{*} P<0.05$. (PDF $99 \mathrm{~kb}$ )

Additional file 13: Figure S8. (A-B) The putative binding sites of TFAP2C in ROCK1 and ROCK2 promoters by JASPAR. (C and D) Schematic representation of the promoter regions of ROCK1 and ROCK2 with the putative TFAP2C binding sites through UCSC. (PDF $171 \mathrm{~kb}$ )

Additional file 14: Figure S9. (A and B) Analysis of ROCK1 and ROCK2 promoters physically associated with TFAP2C by using chromatin immunoprecipitation (ChIP) assay in the indicated HCT116 cells. ${ }^{*} P<0.05$. ( $C$ and D) Relative luciferase activity of the indicated promoter vectors in the indicated HCT116 cells. ${ }^{*} P<0.05$. (PDF $135 \mathrm{~kb}$ )

Additional file 15: Figure S10. (A-D) The specific inhibitor of ROCK1 and ROCK2, Y-27632, significantly repressed SP fraction, sphere formation ability, mitochondrial potential and BCL2, BCL2L1 expression in the TFAP2C-overexpressing CRC cells. (E and F) Representative immunofluorescent images of CRC cells were immunostained with YAP or TAZ antibody (red) or phalloidin (green) in the indicated CRC cells. ( $G$ and $H$ ) The percentage of nuclear TAZ+ $(\mathrm{G})$ and nuclear YAP+ $(\mathrm{H})$ cell number via immunostaining in the indicated groups. ${ }^{*} P<0.05$. (I) Western blotting of ROCK1, ROCK2, p-MST1/2, MST1/2, p-LATS1, LAST1, p-YAP, YAP and TAZ expression, and nuclear YAP and TAZ expression in the indicated cells. atubulin and p84 were used as the loading control for cytoplasm and nucleus respectively. (PDF $383 \mathrm{~kb}$ )

Additional file 16: Figure S11. Clinical relation of TFAP2C with Hippo signaling activity in human CRC tissues. (A) Analysis of TFAP2C expression with $\mathrm{p}-\mathrm{MST} 1 / 2$, $\mathrm{p}-\mathrm{LATS} 1$, YAP and TAZ expression in 4 resistant CRC tissues (T1-4) and 4 sensitive CRC tissues (T5-8). a-tubulin was used as loading control. (B-F) Relative expression levels of TFAP2C, p-MST1/2, p-LATS1, YAP and TAZ expression in CRC tissues.The expression levels of TFAP2C, p-MST1/2, p-LATS1, YAP and TAZ expression were quantified by densitometry using Image J, and normalized to the levels of a-tubulin respectively. The sample with the lowest expression of each protein was used as a standard. (PDF $191 \mathrm{~kb}$ )

\section{Abbreviations}

BMI1: BMI1 Proto-Oncogene, Polycomb Ring Finger; CTGF: Connective Tissue Growth Factor; CYR61: Cysteine Rich Angiogenic Inducer 61; HOXA1: Homeobox A1; IHC: Immunohistochemistry; NANOG: Nanog Homeobox; OCT4: POU Class 5 Homeobox 1; PCR: Polymerase Chain Reaction; ROCK1/2: Rho Associated Coiled-Coil Containing Protein Kinase 1/2; SOX2: SRY-BOX 2; SOX9: SRY-Box 9; TAZ: WW Domain Containing Transcription Regulator 1; TCGA: The Cancer Genome Atlas; TEAD: TEA Domain Transcription Factor; TFAP2C: Transcription Factor AP-2 Gamma; YAP: Yes Associated Protein 1

\section{Acknowledgements}

Not applicable

Ethical approval and consent to participate

The ethics approval statements for animal work were provided by The Institutional Animal Care and Use Committee of Sun Yat-Sen University 
Cancer Center. The ethics approval number for animal work was L102012016110D.

\section{Funding}

This study was supported by the Finance Department Foundation of Jilin Province (3D5157423428).

\section{Availability of data and materials}

The datasets generated and/or analysed during the current study are available in TCGA (TCGA website: https://cancergenome.nih.gov/). Gene Set Enrichment Analysis (GSEA) was performed using GSEA 2.2.1 (http:// www.broadinstitute.org/gsea) and gene set was performed by Molecular Signatures Database v5.2 (http://software.broadinstitute.org/gsea/msigdb)

\section{Authors' contributions}

Lei Wang developed ideas and drafted the manuscript. Xu Wang and Di Sun conducted the experiments and contributed to the analysis of data. Jiandong Tai contributed to the analysis of data. Si Chen and Dong Ren contributed to the analysis of data and revised the manuscript. Miao Yu edited the manuscript. All authors contributed to revise the manuscript and approved the final version for publication.

\section{Consent for publication}

Not applicable.

\section{Competing interests}

The authors declare that they have no competing interests.

\section{Publisher's Note}

Springer Nature remains neutral with regard to jurisdictional claims in published maps and institutional affiliations.

\section{Author details}

${ }^{1}$ Department of Colorectal and Anal Surgery, The First Hospital of Jilin University, 71 Xinmin Street, Changchun, Jilin 130000, People's Republic of China. ${ }^{2}$ Center for Private Medical Service and Healthcare, The First Hospital of Sun Yat-sen University, Guangzhou, Guangdong 510080, China. ${ }^{3}$ Department of Orthopaedic Surgery, The First Affiliated Hospital of Sun Yat-sen University, Guangzhou 510080, China.

\section{Received: 25 October 2017 Accepted: 19 January 2018}

\section{Published online: 13 February 2018}

\section{References}

1. Jemal A, Siegel R, Ward E, Hao Y, Xu J, Thun MJ. Cancer statistics, 2009. CA Cancer J Clin. 2009;59(4):225-49.

2. Ricci-Vitiani L, Fabrizi E, Palio E, De Maria R. Colon Cancer stem cells. J Mol Med. 2009:87(11):1097-104

3. Giacchetti S, Perpoint B, Zidani R, Le Bail N, Faggiuolo R, Focan C, Chollet P, Llory JF, Letourneau Y, Coudert B, et al. Phase III multicenter randomized trial of oxaliplatin added to chronomodulated fluorouracil-leucovorin as first-line treatment of metastatic colorectal cancer. J clin oncol. 2000;18(1):136-47.

4. Douillard JY, Cunningham D, Roth AD, Navarro M, James RD, Karasek P, Jandik P, Iveson T, Carmichael J, Alakl M, et al. Irinotecan combined with fluorouracil compared with fluorouracil alone as first-line treatment for metastatic colorectal cancer: a multicentre randomised trial. Lancet. 2000; 355(9209):1041-7.

5. Singh SK, Hawkins C, Clarke ID, Squire JA, Bayani J, Hide T, Henkelman RM, Cusimano MD, Dirks PB. Identification of human brain tumour initiating cells. Nature. 2004;432(7015):396-401.

6. Park EK, Lee JC, Park JW, Bang SY, Yi SA, Kim BK, Park JH, Kwon SH, You JS, Nam SW, et al. Transcriptional repression of cancer stem cell marker CD133 by tumor suppressor p53. Cell Death Dis. 2015;6:e1964.

7. Longley DB, Johnston PG. Molecular mechanisms of drug resistance J Pathol. 2005;205(2):275-92.

8. Ge G, Zhou C, Ren Y, Tang X, Wang K, Zhang W, Niu L, Zhou Y, Yan Y, He J. Enhanced SLC34A2 in breast cancer stem cell-like cells induces chemotherapeutic resistance to doxorubicin via SLC34A2-Bmi1-ABCC5 signaling. Tumour biol. 2016;37(4):5049-62.

9. Zhang L, Sun H, Zhao F, Lu P, Ge C, Li H, Hou H, Yan M, Chen T, Jiang G, et al. BMP4 administration induces differentiation of CD133+ hepatic cancer stem cells, blocking their contributions to hepatocellular carcinoma. Cancer Res. 2012;72(16):4276-85.

10. Eckert $D$, Buhl $S$, Weber $S$, Jager $R$, Schorle $H$. The AP-2 family of transcription factors. Genome Biol. 2005;6(13):246

11. Schorle H, Meier P, Buchert M, Jaenisch R, Mitchell PJ. Transcription factor AP-2 essential for cranial closure and craniofacial development. Nature. 1996;381(6579):235-8.

12. Zhang J, Hagopian-Donaldson S, Serbedzija G, Elsemore J, Plehn-Dujowich D, AP MM, Flavell RA, Williams T. Neural tube, skeletal and body wall defects in mice lacking transcription factor AP-2. Nature. 1996;381(6579):238-41.

13. Shi D, Xiao X, Tian Y, Qin L, Xie F, Sun R, Wang J, Li W, Liu T, Xiao Y, et al. Activating enhancer-binding protein-2alpha induces cyclooxygenase-2 expression and promotes nasopharyngeal carcinoma growth. Oncotarget. 2015;6(7):5005-21.

14. Fu L, Shi K, Wang J, Chen W, Shi D, Tian Y, Guo W, Yu W, Xiao X, Kang T, et al. TFAP2B overexpression contributes to tumor growth and a poor prognosis of human lung adenocarcinoma through modulation of ERK and VEGF/PEDF signaling. Mol Cancer. 2014;13:89.

15. Berlato C, Chan KV, Price AM, Canosa M, Scibetta AG, Hurst HC. Alternative TFAP2A isoforms have distinct activities in breast cancer. Breast cancer res. 2011:13(2):R23.

16. De Andrade JP, Park JM, Gu WW, Woodfield GW, Kulak MV, Lorenzen AW, Wu VT, Van Dorin SE, Spanheimer PM, Weigel RJ. EGFR is regulated by TFAP2C in luminal breast cancer and is a target for Vandetanib. Mol Cancer Ther. 2016;15(3):503-11.

17. Ebert MP, Tanzer M, Balluff B, Burgermeister E, Kretzschmar AK, Hughes DJ, Tetzner R, Lofton-Day C, Rosenberg R, Reinacher-Schick AC, et al. TFAP2E-DKK4 and chemoresistance in colorectal cancer. N Engl J Med. 2012;366(1):44-53.

18. Pan D. The hippo signaling pathway in development and cancer. Dev Cell. 2010:19(4):491-505.

19. Halder G, Johnson RL. Hippo signaling: growth control and beyond. Development. 2011;138(1):9-22.

20. Harvey KF, Zhang $X$, Thomas DM. The hippo pathway and human cancer. Nat Rev Cancer. 2013;13(4):246-57.

21. Lei QY, Zhang H, Zhao B, Zha ZY, Bai F, Pei XH, Zhao S, Xiong Y, Guan KL. TAZ promotes cell proliferation and epithelial-mesenchymal transition and is inhibited by the hippo pathway. Mol Cell Biol. 2008;28(7):2426-36.

22. Oka T, Mazack V, Sudol M: Mst2 and Lats kinases regulate apoptotic function of yes kinase-associated protein (YAP). J Biol Chem 2008, 283(41): 27534-27546.

23. Zhang H, Liu CY, Zha ZY, Zhao B, Yao J, Zhao S, Xiong Y, Lei QY, Guan $\mathrm{KL}$. TEAD transcription factors mediate the function of TAZ in cell growth and epithelial-mesenchymal transition. J Biol Chem. 2009; 284(20):13355-62

24. Lu L, Li Y, Kim SM, Bossuyt W, Liu P, Qiu Q, Wang Y, Halder G, Finegold MJ, Lee JS, et al. Hippo signaling is a potent in vivo growth and tumor suppressor pathway in the mammalian liver. Proc Natl Acad Sci U S A. 2010; 107(4):1437-42.

25. Imajo M, Miyatake K, limura A, Miyamoto A, Nishida E. A molecular mechanism that links hippo signalling to the inhibition of Wnt/beta-catenin signalling. EMBO J. 2012;31(5):1109-22.

26. Chen M, Wang M, Xu S, Guo X, Jiang J. Upregulation of miR-181C contributes to chemoresistance in pancreatic cancer by inactivating the hippo signaling pathway. Oncotarget. 2015;6(42):44466-79.

27. Touil $Y$, Igoudjil W, Corvaisier M, Dessein AF, Vandomme J, Monte D, Stechly L, Skrypek N, Langlois C, Grard G, et al. Colon Cancer cells escape 5FU chemotherapy-induced cell death by entering stemness and quiescence associated with the c-yes/YAP axis. Clin cancer res. 2014;20(4):837-46.

28. Ren D, Yang Q, Dai Y, Guo W, Du H, Song L, Peng X. Oncogenic miR-210-3p promotes prostate cancer cell EMT and bone metastasis via NF-kappaB signaling pathway. Mol Cancer. 2017;16(1):117.

29. Dai Y, Ren D, Yang Q, Cui Y, Guo W, Lai Y, Du H, Lin C, Li J, Song L, et al. The TGF-beta signalling negative regulator PICK1 represses prostate cancer metastasis to bone. Br J Cancer. 2017;117(5):685-94. https://doi.org/10.1038/ bjc.2017.212. PMID: 28697177.

30. Ren D, Wang M, Guo W, Zhao X, Tu X, Huang S, Zou X, Peng X. Wild-type p53 suppresses the epithelial-mesenchymal transition and stemness in PC-3 prostate cancer cells by modulating miR145. Int J Oncol. 2013;42(4):1473-81.

31. Zhang X, Ren D, Guo L, Wang L, Wu S, Lin C, Ye L, Zhu J, Li J, Song L, et al. Thymosin beta 10 is a key regulator of tumorigenesis and metastasis and a novel serum marker in breast cancer. Breast cancer res. 2017;19(1):15. 
32. Li X, Liu F, Lin B, Luo H, Liu M, Wu J, Li C, Li R, Zhang X, Zhou K, et al. miR150 inhibits proliferation and tumorigenicity via retarding G1/S phase transition in nasopharyngeal carcinoma. Int J Oncol. 2017. https://doi.org/10. 3892/ijo.2017.3909. PMID: 28350089.

33. Wang M, Ren D, Guo W, Huang S, Wang Z, Li Q, Du H, Song L, Peng X. Ncadherin promotes epithelial-mesenchymal transition and cancer stem cell-like traits via ErbB signaling in prostate cancer cells. Int J Oncol. 2016;48(2):595-606.

34. Ren D, Lin B, Zhang X, Peng Y, Ye Z, Ma Y, Liang Y, Cao L, Li X, Li R, et al. Maintenance of cancer stemness by miR-196b-5p contributes to chemoresistance of colorectal cancer cells via activating STAT3 signaling pathway. Oncotarget. 2017;8(30):49807-49823.https://doi.org/10.18632/ oncotarget.17971. PMID: 28591704.

35. Goodell MA. Stem cell identification and sorting using the Hoechst 33342 side population (SP). Curr protoc cytom. 2005; Chapter 9:Unit9 18. https:// doi.org/10.1002/0471142956.cy0918s34. PMID: 18770827.

36. Ren D, Wang M, Guo W, Huang S, Wang Z, Zhao X, Du H, Song L, Peng X. Double-negative feedback loop between ZEB2 and miR-145 regulates epithelial-mesenchymal transition and stem cell properties in prostate cancer cells. Cell Tissue Res. 2014;358(3):763-78.

37. Guo W, Ren D, Chen X, Tu X, Huang S, Wang M, Song L, Zou X, Peng X. HEF1 promotes epithelial mesenchymal transition and bone invasion in prostate cancer under the regulation of microRNA-145. J Cell Biochem. 2013;114(7):1606-15.

38. Zhang X, Zhang L, Lin B, Chai X, Li R, Liao Y, Deng X, Liu Q, Yang W, Cai Y, et al. Phospholipid Phosphatase 4 promotes proliferation and tumorigenesis, and activates Ca2+-permeable Cationic Channel in lung carcinoma cells. Mol Cancer. 2017;16(1):147.

39. Kono K, Tamashiro DA, Alarcon VB. Inhibition of RHO-ROCK signaling enhances ICM and suppresses TE characteristics through activation of hippo signaling in the mouse blastocyst. Dev Biol. 2014;394(1):142-55.

40. Kang J, Kim W, Lee S, Kwon D, Chun J, Son B, Kim E, Lee JM, Youn H, Youn B. TFAP2C promotes lung tumorigenesis and aggressiveness through miR-183and miR-33a-mediated cell cycle regulation. Oncogene. 2017;36(11):1585-96.

41. Ikram F, Ackermann S, Kahlert Y, Volland R, Roels F, Engesser A, Hertwig F, Kocak H, Hero B, Dreidax D, et al. Transcription factor activating protein 2 beta (TFAP2B) mediates noradrenergic neuronal differentiation in neuroblastoma. Mol Oncol. 2016;10(2):344-59.

42. Pellikainen JM, Kosma VM. Activator protein-2 in carcinogenesis with a specia reference to breast cancer-a mini review. Int J Cancer. 2007;120(10):2061-7.

43. Williams CM, Scibetta AG, Friedrich JK, Canosa M, Berlato C, Moss CH, Hurst HC. AP-2gamma promotes proliferation in breast tumour cells by direct repression of the CDKN1A gene. EMBO J. 2009;28(22):3591-601.

44. Gee JM, Eloranta JJ, Ibbitt JC, Robertson JF, Ellis IO, Williams T, Nicholson RI, Hurst HC. Overexpression of TFAP2C in invasive breast cancer correlates with a poorer response to anti-hormone therapy and reduced patient survival. J Pathol. 2009;217(1):32-41.

45. Penna E, Orso F, Cimino D, Tenaglia E, Lembo A, Quaglino E, Poliseno L, Haimovic A, Osella-Abate S, De Pitta C, et al. microRNA-214 contributes to melanoma tumour progression through suppression of TFAP2C. EMBO J. 2011;30(10):1990-2007.

46. Lin CY, Chao A, Wang TH, Lee LY, Yang LY, Tsai CL, Wang HS, Lai CH. Nucleophosmin/B23 is a negative regulator of estrogen receptor alpha expression via AP2gamma in endometrial cancer cells. Oncotarget. 2016; 7(37):60038-52.

47. Bogachek MV, Chen Y, Kulak MV, Woodfield GW, Cyr AR, Park JM, Spanheimer PM, Li Y, Li T, Weigel RJ. Sumoylation pathway is required to maintain the basal breast cancer subtype. Cancer Cell. 2014;25(6):748-61.

48. Zhou D, Conrad C, Xia F, Park JS, Payer B, Yin Y, Lauwers GY, Thasler W, Lee JT, Avruch J, et al. Mst1 and Mst2 maintain hepatocyte quiescence and suppress hepatocellular carcinoma development through inactivation of the Yap1 oncogene. Cancer Cell. 2009;16(5):425-38.

49. Ren A, Yan G, You B, Sun J. Down-regulation of mammalian sterile 20-like kinase 1 by heat shock protein 70 mediates cisplatin resistance in prostate cancer cells. Cancer Res. 2008:68(7):2266-74.

50. Kawahara M, Hori T, Chonabayashi K, Oka T, Sudol M, Uchiyama T. Kpm/ Lats2 is linked to chemosensitivity of leukemic cells through the stabilization of p73. Blood. 2008;112(9):3856-66.

51. Zhang X, George J, Deb S, Degoutin JL, Takano EA, Fox SB, group AS, Bowtell DD, Harvey KF. The hippo pathway transcriptional co-activator, YAP, is an ovarian cancer oncogene. Oncogene. 2011;30(25):2810-22.
52. Overholtzer M, Zhang J, Smolen GA, Muir B, Li W, Sgroi DC, Deng CX, Brugge JS, Haber DA. Transforming properties of YAP, a candidate oncogene on the chromosome 11q22 amplicon. Proc Natl Acad Sci U S A. 2006;103(33):12405-10.

53. Lai $D$, Ho KC, Hao Y, Yang X. Taxol resistance in breast cancer cells is mediated by the hippo pathway component TAZ and its downstream transcriptional targets Cyr61 and CTGF. Cancer Res. 2011;71(7):2728-38.

54. Cao Z, Carey TS, Ganguly A, Wilson CA, Paul S, Knott JG. Transcription factor AP-2gamma induces early Cdx2 expression and represses HIPPO signaling to specify the trophectoderm lineage. Development. 2015;142(9):1606-15.

55. Powe DG, Akhtar G, Habashy HO, Abdel-Fatah T, Rakha EA, Green AR, Ellis IO. Investigating AP-2 and YY1 protein expression as a cause of high HER2 gene transcription in breast cancers with discordant HER2 gene amplification. Breast cancer res. 2009;11(6):R90.

\section{Submit your next manuscript to BioMed Central and we will help you at every step:}

- We accept pre-submission inquiries

- Our selector tool helps you to find the most relevant journal

- We provide round the clock customer support

- Convenient online submission

- Thorough peer review

- Inclusion in PubMed and all major indexing services

- Maximum visibility for your research

Submit your manuscript at www.biomedcentral.com/submit
) Biomed Central 